\title{
Escherichia coli Resistance to Ciprofloxacin in Acute Uncomplicated Pyelonephritis
}

\author{
Emad Abu Sitta, Nour Aljariri Alhesan, Babikir Kheiri, Sunil Badami, Farah Elounais, \\ Omar Assassa, Maneesh Gaddam, Carlos Rios-Bedoya \\ Hurley Medical Center, Michigan State University, East Lansing, USA \\ Email: eabusit1@hurleymc.com, naljari1@hurleymc.com, babikir.kheiri@hotmail.com, sbadami1@hurleymc.com, \\ felouna1@hurleymc.com, oassasa1@hurleymc.com, mgaddam1@hurleymc.com,crios1@hurleymc.com
}

How to cite this paper: Sitta, E.A., Alhesan, N.A., Kheiri, B., Badami, S., Elounais, F., Assassa, O., Gaddam, M. and Rios-Bedoya, C. (2017) Escherichia coli Resistance to Ciprofloxacin in Acute Uncomplicated Pyelonephritis. Open Access Library Journal, 4: e4043.

https://doi.org/10.4236/oalib.1104043

Received: October 19, 2017

Accepted: November 10, 2017

Published: November 13, 2017

Copyright $\odot 2017$ by authors and Open Access Library Inc.

This work is licensed under the Creative Commons Attribution International License (CC BY 4.0).

http://creativecommons.org/licenses/by/4.0/

\begin{abstract}
Background: Severe acute uncomplicated pyelonephritis is an infection of the kidneys that usually have an ascending route and occur in presumably healthy urinary tract. The most common pathogen involved is $E$. coli. The Infectious Diseases Society of America (IDSA) has issued an updated guideline in 2010 suggesting IV quinolones to be considered in the initial empiric antimicrobial therapy giving known resistance of less than $10 \%$. However, E. coli resistance to quinolones has been increasing, the recent data of $E$. coli, causing pyelonephritis, resistance is not known in the Midwest. Local hospital antibiogram for two years showed up to $22 \%$ resistance to ciprofloxacin among $E$. coli isolates. Methods: We conduct a retrospective non-concurrent cohort study in one teaching hospital in the Midwest, females who were admitted with severe acute uncomplicated pyelonephritis in a three years period were included. Patients with urinary tract obstruction, pregnancy, immuno-suppression, males, and indwelling Foley's catheters were excluded. Data collected include causative pathogens and resistance to antibiotics were collected. Percentages, frequencies, and measures of central tendency and dispersion were calculated to describe the study sample Results: 73 patients were included in the final analysis. E. coli was the most common isolated pathogen ( $81 \%)$, followed by other enteric gram negative. E. coli resistance to ciprofloxacin was $13.5 \%, 37 \%$ to trimethoprim-sulfamethoxazole, and $5 \%$ to ceftriaxone. Conclusion: Ciprofloxacin should be avoided initially in treating severe acute uncomplicated pyelonephritis until culture results and sensitivity is available.
\end{abstract}

\section{Subject Areas}

Pharmacology 


\section{Keywords}

E. coli, Pyelonephritis, Ciprofloxacin

\section{Introduction}

Acute pyelonephritis is a severe infection of the kidney, usually have an ascending route of infection, that carries an annual incidence of 15 - 17 cases per 10,000 population among females [1]. It is classified into complicated and uncomplicated pyelonephritis, where complicated urinary tract infection (UTI) occurs in patients with abnormal urinary tract. UTI and pyelonephritis are considered uncomplicated when it occurs in non-pregnant females and presumed healthy urinary tract [2] [3]. E. coli is the most common cause of uncomplicated pyelonephritis accounting for almost $80 \%$, followed by other enteric gram negative bacteria [1] [3].

The Infectious Diseases Society of America (IDSA) has issued in 2010 a clinical practice guideline for the treatment of uncomplicated cystitis and pyelonephritis in women. It recommends the use of intravenous antimicrobial, such as fluoroquinolones; an aminoglycoside; an extended-spectrum cephalosporin or extended-spectrum penicillin; or carbapenem [2]. They also suggest considering the local $E$. coli antimicrobial resistance when selecting the initial intravenous antimicrobial, resistance prevalence threshold above which the fluoroquinolones is not recommended is $10 \%$ [3]. Rates of quinolones resistance among $E$. coli have shown an upward trend [4]. Consequently, IDSA has suggested continuing monitoring local E. coli resistance and evaluating its rate over time to sustain optimum empiric therapy [2].

According to our knowledge, local data for $E$. coli resistance is missing in the Midwest, it has been reported that United State medical centers have higher rates of resistance than Canadian centers. This study was performed at a community hospital in the Midwest, the hospital's microbiology antibiogram for 2016 has shown 20\% in vitro ciprofloxacin resistance among E. coli isolates (in 2015 it was $22 \%$ ). Ceftriaxone resistance was only 5\%; this antibiogram reported all $E$. coli isolates from all sources (blood, urine, skin, etc.) and doesn't differentiate between clinical infection or colonization.

The aim of this study is to follow the E. coli resistance trend in the Midwest following the IDSA recommendation.

\section{Methods}

Setting: A 443-bed public teaching hospital located in the Midwest.

Subjects: This study included all female patients who were admitted to the hospital between January 2013 and December 2016 with acute uncomplicated pyelonephritis. Patients were identified from the electronic medical records using the international classification of diseases-9 (ICD-9) and 10 (ICD-10). Codes 
for pyelonephritis, sepsis, and urinary tract infection were used. The study was reviewed and approved by the cognizant Institutional Review Board.

Project Design: This is a non-concurrent cohort study comprised of 362 patients whose charts were reviewed. Patients with complicated pyelonephritis were excluded (pregnant, males, urinary tract obstruction by stones or tumors, immune-compromised, indwelling foleys catheter, urinary incontinence, advanced uncontrolled diabetes mellitus, and patients with ureteral stents); patients with simple cystitis were excluded as well. Only patients with positive urine culture were included. A total 72 patients met the inclusion criteria for this study.

Data-Collection: Sociodemographic, pyelonephritis clinical features, and laboratory and microbiological information were obtained from medical charts using the electronic medical records system. Data gathered included patient's age, race, presence or absence of co-morbidities (e.g. diabetes mellitus, hypertension, chronic kidney disease, and non-obstructing kidney stones), laboratory studies, blood culture results, urine culture results, initial antibiotic used, complications, (e.g. Clostridium difficile infection), and health outcomes (e.g. in-hospital death).

Data-Analysis: Before any statistical analysis was done, we determined the presence of data outliers, out of range values, and any need for data cleaning and editing by performing a series of frequencies, proportions, descriptive statistics (e.g. mean, median and standard deviation) and figures (e.g. histograms and box and whisker plots). After this process was completed, any needed data editing and cleaning was conducted. Percentages, frequencies, and measures of central tendency and dispersion were calculated to describe the study sample. All analyses were done using Stata (Stata Corporation, College Station, TX).

\section{Results}

A total of 72 patients met the criteria for analysis; selected sociodemographic characteristics, laboratory findings, outcomes, and co-morbidities are summarized in Table 1. Mean age was 35.2 years, $22 \%$ of the patients are diabetic, and these patients have controlled diabetes mellitus and has no genitourinary complications. Patients with neurogenic bladder as a complication from diabetes mellitus were excluded.

Causative bacteria are summarized in Table 2. Most common organism was Escherichia coli, followed by Klebsiella pneumonia. This is consistent with the known causative agents for urinary tract infections in general.

Resistance pattern of $E$. coli is summarized in Table 3, $5 \%$ of the isolates were ESBL positive. Ceftriaxone was chose as the first empirical antibiotics in $58 \%$ of the cases while ciprofloxacin in $30.5 \%$. Among E. coli isolates, $13.5 \%$ were resistant to Ciprofloxacin.

There was no in-hospital deaths or development of CD colitis.

\section{Discussion}

Acute uncomplicated pyelonephritis that is severe enough requiring admission 
Table 1. Patient characteristics, laboratory finding, and outcome.

\begin{tabular}{cc}
\hline Characteristics & \\
\hline Mean Age (years) & 35.2 \\
African American & $54 \%$ \\
Diabetes Mellitus & $22 \%$ \\
Hypertension & $30 \%$ \\
Chronic Kidney Disease & $7 \%$ \\
Non-Obstructing kidney stone & $13 \%$ \\
White Blood cells > 10 K & $57 \%$ \\
Urine Leukocyte esterase & $83 \%$ \\
Pyuria & $95 \%$ \\
Urine Nitrates & $44 \%$ \\
Intensive care unit admission & $6 \%$ \\
Septic shock & $4 \%$ \\
Acute Kidney Injury & $13 \%$ \\
\hline
\end{tabular}

Table 2. Causative bacteria.

\begin{tabular}{cc}
\hline Bacteria & (n) \\
\hline Escherichia coli & $82.0 \%(59)$ \\
Klebsiella pneumoniae & $14.0 \%(10)$ \\
Citrobacter & $2.7 \%(2)$ \\
Pseudomonas aeruginosa & $1.3 \%(1)$ \\
\hline
\end{tabular}

Table 3. Antibiotics resistance among E. coli.

\begin{tabular}{cc}
\hline Antibiotics & Resistance percentage (\%) \\
\hline Ceftriaxone & 5.0 \\
Cefazolin & 17.0 \\
Ciprofloxacin & 13.5 \\
Cefepime & 5.0 \\
Piperacillin-tazobactam & 1.6 \\
Bactrim & 37.0 \\
Imipenem & 0.0 \\
\hline
\end{tabular}

to the hospital and intravenous antibiotics can result in significant morbidity and possibly mortality if not treated properly with the right empiric antibiotic. Choosing the best antibiotics should be based on the recent guidelines and guided by the local data of bacterial resistance, it is also important to take into consideration the clinical condition of the patient. Local epidemiological data is not readily available in all settings, and some hospitals depend on microbiology laboratory derived bacterial resistant chart (antibiogram). The antibiogram pro- 
vide guidance keeping in mind the accuracy is not optimum as these data represents all isolates and not specific for specific diseases, one study compared one hospital antibiogram with actual cystitis cases and found less E. coli resistance in cystitis patients than what was reported in the antibiogram [5].

$E$. coli is the most common causative pathogen in uncomplicated pyelonephritis as well as UTI (75\% - 90\%) [1] [6]. Our study showed that $E$. coli is responsible for $81 \%$ of the cases. Quinolones and Ciprofloxacin are widely used in treating uncomplicated pyelonephritis and considered to be highly effective for inpatient and outpatient management [7]. In our study, $30 \%$ of patients received Ciprofloxacin as the initial antibiotics. Some E. coli strains can develop resistance to ciprofloxacin through either changes in the targeted protein or decreased penetration, and that is acquired through chromosomal mutation [8] [9]. Recent studies has suggested an increasing trend of Cipro resistance among E. coli isolates [10]. IDSA and some recent review articles has suggested a threshold of 10\% Cipro resistance above which Quinolones should not be used empirically before the sensitivity results [2] [3]. Our study found $13.5 \%$ resistance among ciprofloxacin in E. coli isolates in female patients admitted with severe acute uncomplicated pyelonephritis, this increasing resistance maybe due to increasing usage of quinolones in general treating the simple cystitis. It was also noted that $E$. coli has high resistance to trimethoprim-sulfamethoxazole (37\%). This is higher than the previously reported resistance range from previous studies (up to 22\%) [10].

This study suggests using other antibiotics than ciprofloxacin and trimethoprimsulfamethoxazole that are known to be effective empirically until culture results are back in treating severe uncomplicated pyelonephritis inpatient. Weather these results should be used in treating mild to moderate cases of pyelonephritis is uncertain, additional studies are required to determine valid resistance patterns.

\section{Limitations}

Our study included only patients with severe pyelonephritis requiring inpatient treatment. The reported E. coli resistance could be different in less severe cases of pyelonephritis.

\section{Conclusion}

Ciprofloxacin and Trimethoproim-sulfamethaxazole should be avoided as first line empiric antibiotics for severe cases of uncomplicated pyelonephritis in this Midwest teaching hospital.

\section{References}

[1] Czaja, C.A., Scholes, D., Hooton, T.M., et al. (2007) Population-Based Epidemiologic Analysis of Acute Pyelonephritis. Clinical Infectious Diseases, 45, 273-280. https://doi.org/10.1086/519268

[2] Gupta, K., Hooton, T.M., Naber, K.G., et al. (2011) International Clinical Practice 
Guidelines for the Treatment of Acute Uncomplicated Cystitis and Pyelonephritis in Women: A 2010 Update by the Infectious Diseases Society of America and the European Society for Microbiology and Infectious Diseases. Clinical Infectious Diseases, 52, e103-e120.

[3] Hooton, T.M. (2012) Uncomplicated Urinary Tract Infection. New England Journal of Medicine, 366, 1028-1037. https://doi.org/10.1056/NEJMcp1104429

[4] Zhanel, G., Hisanaga, T., Laing, N., et al. (2006) Antibiotic Resistance in Escherichia coli Outpatient Urinary Isolates: Final Results from the North American Urinary Tract Infection Collaborative Alliance (NAUTICA). International Journal of Antimicrobial Agents, 27, 468-475. http://www.ncbi.nlm.nih.gov/pubmed/16713191 https://doi.org/10.1016/j.ijantimicag.2006.02.009

[5] Smith, S.C., Bazzoli, C., Chung, I., et al. (2015) Antimicrobial Susceptibility of Escherichia coli in Uncomplicated Cystitis in the Emergency Department: Is the Hospital Antibiogram an Effective Treatment Guide? In: Bogucki, S., Ed., Academic Emergency Medicine, 22, 998-1000.

http://www.ncbi.nlm.nih.gov/pubmed/26250654

https://doi.org/10.1111/acem.12729

[6] Echols, R.M., Tosiello, R.L., Haverstock, D.C., et al. (1999) Demographic, Clinical, and Treatment Parameters Influencing the Outcome of Acute Cystitis. Clinical Infectious Diseases, 29, 113-119. https://doi.org/10.1086/520138

[7] Talan, D.A., Stamm, W.E., Hooton, T.M., et al. (2017) Comparison of Ciprofloxacin (7 Days) and Trimethoprimsulfamethoxazole (14 Days) for Acute Uncomplicated Pyelonephritis Pyelonephritis in Women: A Randomized Trial. JAMA, 283, 1583 1590. http://www.ncbi.nlm.nih.gov/pubmed/10735395

[8] Hooper, D.C. (1999) Mechanisms of Fluoroquinolone Resistance. Drug Resistance Updates, 2, 38-55. http://linkinghub.elsevier.com/retrieve/pii/S1368764698900681 https://doi.org/10.1054/drup.1998.0068

[9] Martínez-Martínez, L., Pascual, A. and Jacoby, G.A. (1998) Quinolone Resistance from a Transferable Plasmid. Lancet (London, England), 351, 797-799. http://linkinghub.elsevier.com/retrieve/pii/S0140673697073224 https://doi.org/10.1016/S0140-6736(97)07322-4

[10] Sanchez, G.V., Master, R.N., Karlowsky, J.A., et al. In Vitro Antimicrobial Resistance of Urinary Escherichia coli Isolates among U.S. Outpatients from 2000 to 2010. Antimicrobial Agents and Chemotherapy, 56, 2181-2183. 
Submit or recommend next manuscript to OALib Journal and we will provide best service for you:

- Publication frequency: Monthly

- 9 subject areas of science, technology and medicine

- Fair and rigorous peer-review system

- Fast publication process

- Article promotion in various social networking sites (LinkedIn, Facebook, Twitter, etc.)

- Maximum dissemination of your research work

Submit Your Paper Online: Click Here to Submit

Or Contact service@oalib.com 